\title{
Changes in human cervical and lumbar spine curves while bicycling with different handlebar heights
}

\author{
Yi-Lang Chen and Kuo-Chang He \\ Department of Industrial Engineering and Management, Mingchi University of Technology, New Taipei City, \\ Taiwan, R.O.C.
}

\begin{abstract}
Cycling is a daily activity that needs a high demand of human-bicycle fitness. However, studies into the fitness or ergonomic aspects are very little. In this study, the simulated 20 min bicycling test were performed by 26 male participants under 5 handle height conditions. Body joint angles and external cervical/lumbar spine lordosis were measured at the initial and cycling after $20 \mathrm{~min}$. Results show that different handle heights did cause various trunk inclinations. Trunk inclination was negatively and positively correlated with lumbosacral angle $(\mathrm{r}=-0.620, \mathrm{p}<0.001)$ and cervical angle $(\mathrm{r}=0.510, \mathrm{p}<0.001)$, respectively. In this study, regression models were also developed to predict the internal cervical and lumbar spine movements by external trunk inclination and head extension, respectively. The explanatory abilities for the variance of the models were $67.2 \%$ for LSA and $82.8 \%$ for CE prediction. This can be used to understand the cyclist's spine movements while field study of bicycling.
\end{abstract}

Keywords: cycling, handle height, spinal postures, predictive model

\section{Introduction}

Cycling is a daily activity that needs a high demand of human-bicycle fitness. Previous studies have focused on biomechanical analyses (Shan 2008), physiological response (De Bruyne et al. 2010), and kinematics (Kolehmainen et al., 1989), while cycling. Some investigators have also demonstrated that different handle heights change the rider's trunk inclination and indirectly influence the stress on different parts of the rider's body. An individual who cannot feel comfortable while cycling will not ride very long or may become injured. Unfortunately, studies into the bicyclist's spine movement or ergonomic aspects are very little.

\section{Method}

In this study, a simulated 20 min bicycling test were performed by 26 male participants under 5 handlebar height conditions $(16,8,0,-8,-16 \mathrm{~cm}$, those were horizontally referred to the saddle). The mean (SD) age, stature, and body weight was 22.2 (2.0) years, $171.3(6.2) \mathrm{cm}$, and $66.6(6.7) \mathrm{kg}$ for the participants. Body joint angles and external cervical/lumbar spine lordosis were measured (Qualisys MacReflex, Sweden) at the initial and cycling after $20 \mathrm{~min}$. Six adhesive reflective markers and two stick markers were attached to the participant's body landmarks and skin surfaces of spinal processes, respectively (as shown in Figure 1). Before data collection, each participant was given information about the purpose and procedure of this experiment. The basic and anthropometric data were collected.

In this study, one-way ANOVA was conducted to evaluate the handle height effect on the measured spinal angles. The dependent variables included external lumbosacral angles (ELSA) and trunk angle (TA), cervical extension (CE), and head extension (HE) (Figure 1). Duncan's multiple range test was also used as pos-hoc testing. The ELSAs were then transformed into the internal LSA by the predictive models that 
were developed by Chen and Lee (1997) and are as follows.

$$
\begin{array}{ll}
\mathrm{L} 1=0.9882 \times \mathrm{SL} 1+3.6274 & \left(\mathrm{R}^{2}=0.968\right) \\
\mathrm{S} 1=0.7339 \times \mathrm{SS} 1+29.6776 & \left(\mathrm{R}^{2}=0.916\right)
\end{array}
$$

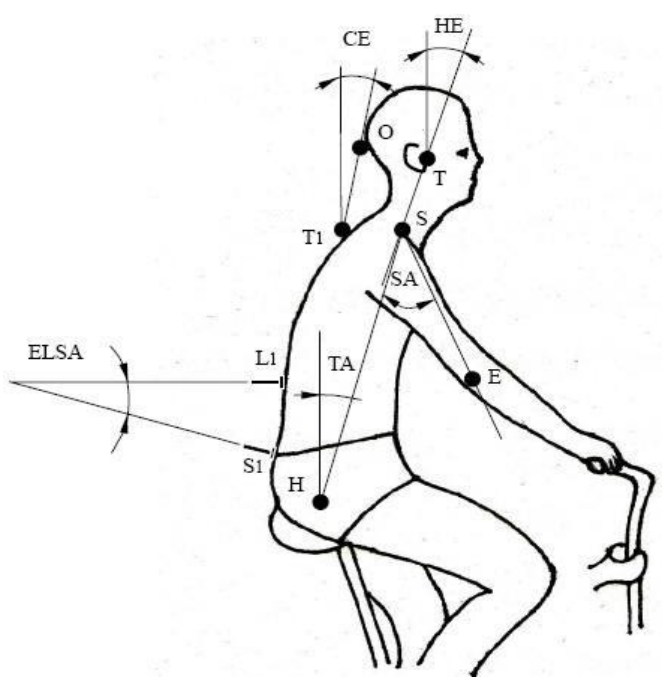

$$
\begin{array}{ll}
\text { TA: trunk angle } & \text { T1: } 1^{\text {st }} \text { thoracic process } \\
\text { ELSA: external LSA } & \text { L1: } 1^{\text {th }} \text { lumbar process } \\
\text { HE: head extension } & \text { S1: } 1^{\text {st }} \text { sacral process } \\
\text { CE: cervical extension } & \text { SA: shoulder angle } \\
\text { T: tragus } & \text { O: external occipital protubernance }
\end{array}
$$

Figure 1. The definition of the body joint angles and spine curves while cycling

\section{Results and discussion}

Results show that different handle heights caused various riding postures, therefore resulted in different cervical and lumbar curvatures. Trunk inclination was negatively and positively correlated with lumbosacral angle $(\mathrm{r}=-0.620, \mathrm{p}<0.001)$ and cervical extension $(\mathrm{r}=$ $0.510, \mathrm{p}<0.001)$, respectively. Table 1 and 2 show that handle heights significantly influenced the cyclist's LSA and CE $(p<0.05)$. The lower handle heights would result in less lumbar lordosis and more cervical extension and therefore cause more stress on the spine. In sum, bicycle with higher bars were recommended when considering the spinal curvatures. In this study, regression models were also developed to predict the internal LSA and CE by trunk angle (TA), head extension (HE), and upper arm length (UAL). The explana- tory abilities for the variance of the models were $67.2 \%$ for LSA and $82.8 \%$ for CE prediction. The models can be used to understand the cyclist's spine movements in a field bicycling test and are as follows.

$$
\begin{array}{ll}
\mathrm{LSA}=0.195 \times \mathrm{HE}-0.519 \times \mathrm{TA}+29.0 & \left(\mathrm{R}^{2}=0.672\right) \\
\mathrm{CE}=1.010 \times \mathrm{HE}+1.730 \times \mathrm{UAL}-59.2 & \left(\mathrm{R}^{2}=0.828\right)
\end{array}
$$

Table 1. ANOVA result for lumbosacral angles (LSA)

\begin{tabular}{cccc}
\hline Handle heights $(\mathrm{cm})$ & $\mathrm{N}$ & LSA $\left({ }^{\circ}\right)$ & Duncan MRT \\
\hline-16 & 26 & $12.9(4.8)^{*}$ & $\mathrm{~A}$ \\
-8 & 26 & $13.9(4.6)$ & $\mathrm{A}$ \\
0 & 26 & $14.5(5.2)$ & $\mathrm{A}$ \\
8 & 26 & $19.0(4.1)$ & $\mathrm{B}$ \\
16 & 26 & $21.6(4.8)$ & $\mathrm{B}$ \\
\hline
\end{tabular}

* mean (SD)

\begin{tabular}{cccc}
\multicolumn{5}{l}{ Table 2. ANOVA result for cervical extension (CE) } \\
\hline Handle heights $(\mathrm{cm})$ & $\mathrm{N}$ & $\mathrm{CE}\left({ }^{\circ}\right)$ & Duncan MRT \\
\hline-16 & 26 & $55.9(8.6)^{*}$ & $\mathrm{~A}$ \\
-8 & 26 & $53.6(7.9)$ & $\mathrm{A}$ \\
0 & 26 & $44.5(8.3)$ & $\mathrm{B}$ \\
8 & 26 & $40.0(9.0)$ & $\mathrm{B}$ \\
16 & 26 & $37.0(7.1)$ & $\mathrm{B}$ \\
\hline
\end{tabular}

* mean (SD)

\section{Conclusions}

The finding shows that bicycle with higher bars were recommended when considering the spinal curvatures. We also developed the predictive models to obtain the cyclist's lumbosacral angle and cervical extension while performing a field cycling test.

\section{References}

[1] Chen, Y. L. and Lee, Y. H., 1997. A non-invasive protocol for the determiniation of lumbosacral vertebral angle. Clinical Biomechanics, 12, 185-189.

[2] De Bruyne, G., Aerts, J. M., Sloten, J. V., Goffin, J., Verpoest, I., Berckmans, D., 2010. Transient sweat response of the human head during cycling. International Journal of Industrial Ergonomics, 40, 406-413.

[3] Kolehmainen, I., Harms-Ringdahl, K., Lanshammar, H., 1989. Cervical spine positions and load moments during bicycling with different handlebar positions. Clinical Biomechanics, 4, 105-110.

[4] Shan, G., 2008. Biomechanical evaluation of bike power saver. Applied Ergonomics, 39, 37-45. 